This item was submitted to Loughborough's Research Repository by the author.

Items in Figshare are protected by copyright, with all rights reserved, unless otherwise indicated.

\title{
Understanding the role of selenium in defect passivation for highly efficient selenium-alloyed cadmium telluride solar cells
}

PLEASE CITE THE PUBLISHED VERSION

https://doi.org/10.1038/s41560-019-0389-z

\section{PUBLISHER}

(c) the authors. Published by Springer

\section{VERSION}

AM (Accepted Manuscript)

\section{PUBLISHER STATEMENT}

This paper was accepted for publication in the journal Nature Energy and the definitive published version is available at https://doi.org/10.1038/s41560-019-0389-z.

\section{LICENCE}

\section{All Rights Reserved}

\section{REPOSITORY RECORD}

Fiducia, Tom, Budhika G. Mendis, Kexue Li, Chris R.M. Grovenor, Amit Munshi, Kurt L. Barth, Walajabad S. Sampath, et al.. 2019. "Understanding the Role of Selenium in Defect Passivation for Highly Efficient Selenium-alloyed Cadmium Telluride Solar Cells". figshare. https://hdl.handle.net/2134/37800. 


\title{
Understanding the Role of Selenium in Defect Passivation for Highly Efficient Selenium- Alloyed Cadmium Telluride Solar Cells
}

Thomas A. M. Fiducia ${ }^{1}$, Budhika G. Mendis ${ }^{2}$, Kexue $^{3}{ }^{3}$, Chris R. M. Grovenor ${ }^{3}$, Amit H. Munshi ${ }^{4}$, Kurt

Barth $^{4}$, Walajabad S. Sampath ${ }^{4}$, Lewis D. Wright ${ }^{1}$, Ali Abbas ${ }^{1}$, Jake W. Bowers ${ }^{1}$, and John M. Walls ${ }^{1}$

${ }^{1}$ Centre for Renewable Energy Systems Technology (CREST), Wolfson School of Mechanical, Electrical and Manufacturing Engineering, Loughborough University, Loughborough, Leicestershire, LE11 3TU, United

Kingdom.

${ }^{2}$ Department of Physics, Durham University, South Road, Durham DH1 3LE, United Kingdom.

${ }^{3}$ Materials Department, Oxford University, Oxford, OX1 3PH, United Kingdom.

${ }^{4}$ Colorado State University, Fort Collins, Colorado, 80523, USA.

\begin{abstract}
Electricity produced by cadmium telluride (CdTe) photovoltaic modules is the lowest cost in the solar industry, and now undercuts fossil fuel-based sources in many regions of the world. This is due to recent efficiency gains brought about by alloying selenium into the CdTe absorber, which has taken cell efficiency from $19.5 \%$ to its current record of $22.1 \%$. While the addition of selenium is known to reduce the bandgap of the absorber material and hence increase cell short-circuit current, this effect alone does not explain the performance improvement. Here, by means of cathodoluminescence (CL) and secondary ion mass spectrometry (SIMS), we show that selenium enables higher luminescence efficiency and longer diffusion lengths in the alloyed material, indicating that selenium passivates critical defects in the bulk of the absorber layer. This passivation effect explains the record-breaking performance of selenium-alloyed CdTe devices, and provides a route for further efficiency improvement that can result in even lower costs for solar generated electricity.
\end{abstract}

\section{Introduction}


In the last 7 years the efficiency of cadmium telluride (CdTe) solar cells has improved from $16.7 \%$ to $22.1 \%$ [1], [2]. This has enabled the cost of CdTe photovoltaic electricity to decrease to the point where it is lower than for silicon photovoltaics, and lower than for conventional fossil fuel sources in many regions of the world [3], [4]. The most recent efficiency improvements, from $19.5 \%$ to $22.1 \%$, have been achieved by the addition of selenium to the front of the CdTe absorber layer [5]. This creates a $\mathrm{CdSe}(\mathrm{x}) \mathrm{Te}(1-\mathrm{x})(\mathrm{CdSeTe})$ alloy that lowers the material bandgap, increases absorption in the long wavelength part of the spectrum, and increases device short-circuit current density [6]-[8]. However, in addition to improved current generation, selenium alloying maintains or improves open circuit voltages - despite the lower bandgap [9]. Recent studies have shown that this is associated with improved minority carrier lifetimes in the absorber, but it is not known why the lifetimes increase [9]-[11].

One reason that has been proposed for the higher carrier lifetimes is that the selenium improves band alignments between the absorber and buffer layers at the front interface of the device, reducing interfacial recombination [9]. Another is that the wider-bandgap, non-alloyed CdTe at the back of the device acts as an electron reflector and reduces back surface recombination [12]. These explanations both relate to the effects of selenium at the device level, i.e. its effects on the one-dimensional band structure through the depth of the cell. However the effects of selenium on the basic optoelectronic properties of the absorber material have not been investigated.

Here we use high resolution cathodoluminescence (CL) microscopy to map nanoscale variations in electronic properties in a high efficiency selenium-graded CdTe device, including variations in luminescence efficiency, diffusion length, and effective bandgap. We then compare these with secondary ion mass spectrometry (SIMS) elemental maps of the selenium distribution from the same area of the film, so that the electronic property variations can be directly related to nanoscale changes in the selenium concentration.

The results reveal that selenium causes clear and dramatic improvements in the local luminescence efficiency of the absorber material, indicating that it passivates deep-level defects in CdTe. This provides an explanation for the superior voltage and performance of selenium-alloyed CdTe devices and may enable further gains in efficiency beyond the current record level of $22.1 \%$.

\section{Selenium diffusion into the CdTe layer}

In order to perform the investigation, a cell stack incorporating a $\sim 1.5 \mu \mathrm{m}$ layer of $\mathrm{CdSeTe}$ at the front of the absorber layer was fabricated as shown schematically in Fig 1a and described in the Methods section. Following a cadmium 
chloride $\left(\mathrm{CdCl}_{2}\right)$ activation treatment, which is used universally to produce high efficiency CdTe-based devices [13][16], the 


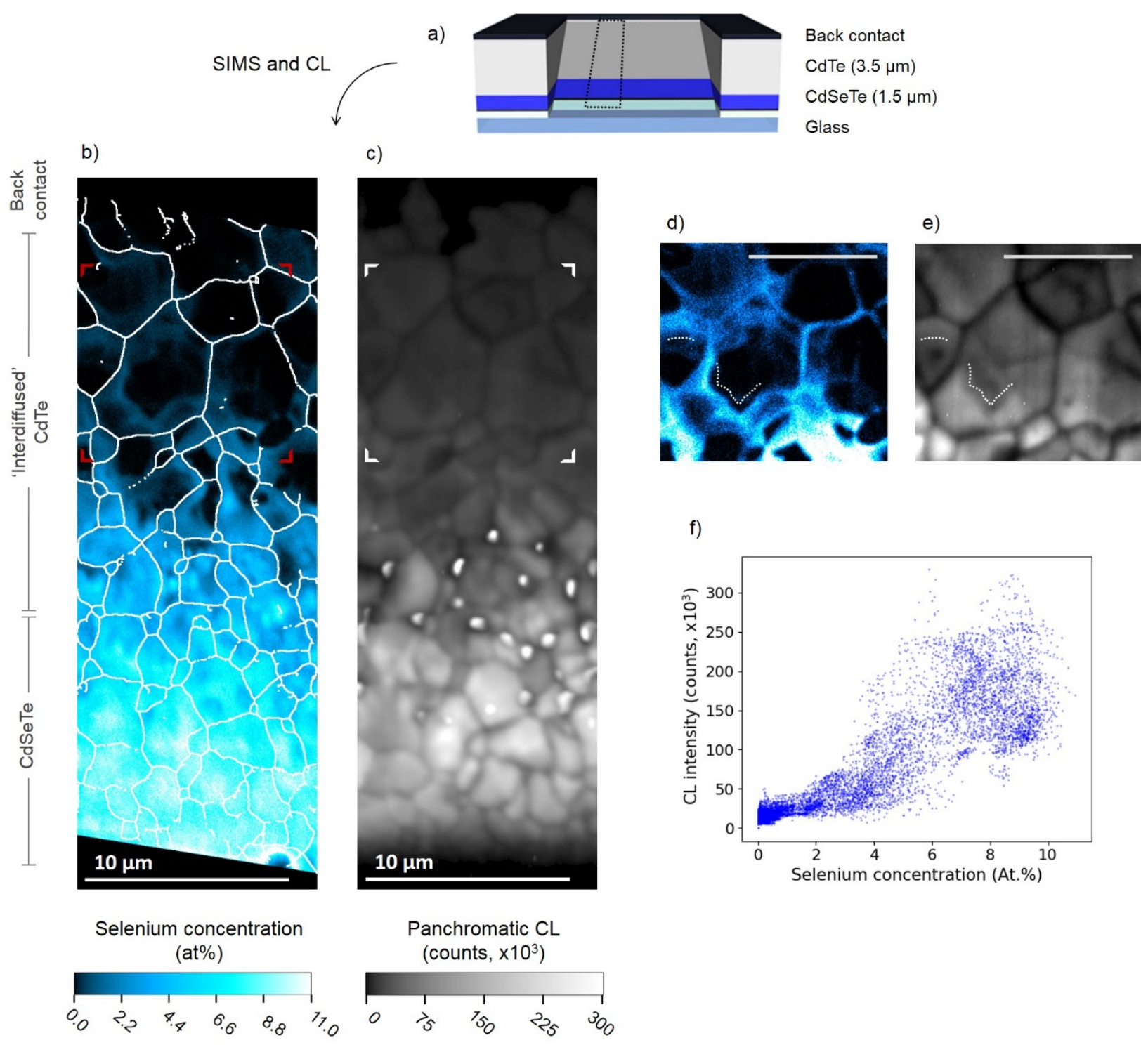

Fig 1: CdTe luminescence efficiency improved by selenium alloying. (a) Schematic of the $\mathrm{CdSeTe} / \mathrm{CdTe}$ device structure and $7^{\circ}$ bevel, with an outline of the SIMS and CL measurement area (dotted black line). (b) SIMS elemental map of the selenium distribution on the bevelled surface of the $\mathrm{CdCl}_{2}$ treated device, with the 'skeletonised' chlorine signal overlaid in white to help delineate grain boundaries (see Methods section for calibration of the selenium concentration). Darker blue corresponds to lower selenium concentrations and brighter cyan/white corresponds to higher concentrations, towards 10 at $\%$ (see colour bar). (c) Cathodoluminescence (CL) map of the panchromatic CL intensity, taken on the same area as the selenium map. Black and dark grey corresponds to lower CL signal intensity 
and brighter white to higher signal intensity (see colour bar). (d) High magnification selenium map of a region at the top of the bevel, shown by the red annotations in (b) (the image has no skeletonised chlorine overlay, so that selenium signal at the grain boundaries can be seen). The scale bar is $5 \mu \mathrm{m}$ ). (e) Corresponding high magnification CL map to the SIMS map in (d). The dashed lines in (d) and (e) highlight similarity in positioning of the selenium and CL signals. (f) Scatter plot of selenium concentration versus CL counts for equivalent regions (i.e. pixels) in (b) and (c), with grain boundary regions and voids omitted from the analysis (voids can be seen as bright white spots in the panchromatic CL in (c)). 
cell was measured to have an efficiency of $16.8 \%$. This value is high amongst CdTe photovoltaics research laboratories, and devices made using this exact deposition system and method have achieved certified conversion efficiencies of up to $18.3 \%$ [17] (only exceeded by the $22.1 \%$ champion cell fabricated by First Solar Inc [2]). After the efficiency measurement, a shallow $7^{\circ}$ bevel was milled through the device stack using a Focussed Ion Beam (FIB). This provided direct access to the CdSeTe layer and presented an extended, smooth cross-section for the CL and elemental mapping. Cathodoluminescence measurements were performed on the bevel surface on an area as outlined on the schematic in Fig 1a. Correlative SIMS elemental mapping at high resolution was then performed on exactly the same region as the $\mathrm{CL}$.

A NanoSIMS map of the selenium concentration on the bevel surface in the $\mathrm{CdCl}_{2}$-treated cell is shown in Fig $1 \mathrm{~b}$. At the bottom of the bevel, near the front interface of the absorber layer, selenium concentrations are in the range 8$10.5 \mathrm{at} \%$ (cyan/white). This is similar to the as-deposited device. However, in contrast to the as-deposited device, which has a sharp interface between the CdSeTe and CdTe layers (see [18], and Supplementary Figure 1), the $\mathrm{CdCl}_{2}$ treated cell shows a gradual decrease in the selenium concentration with distance away from the front interface. This means that selenium signal is detected towards the back of the cell, in the CdTe layer (for clarity now referred to as the 'interdiffused CdTe' region).

In addition to this grading through the absorber depth, there is also some non-uniformity in the selenium concentration around the grain structure of the absorber. In the interdiffused CdTe region at the top of the bevel, close to the back contact, there is an enhanced selenium signal at the grain boundaries and around the fringes of the grains (this can be seen clearly in the high magnification image in Fig 1d). However at the bottom of the bevel, in the CdSeTe region, the selenium concentration is lower around the grain boundaries than in the grain interiors.

This distribution of selenium around grain boundaries indicates that during the high-temperature cadmium chloride activation treatment the grain boundaries provide channels for fast diffusion of selenium from the CdSeTe layer into the CdTe above, which then slowly out-diffuses from the grain boundaries into the grain interiors. This is a mixed grain boundary and lattice diffusion regime of type B in the Harrison classification system, and is also observed with sulphur interdiffusion in conventional CdS/CdTe devices [15], [19]. 


\section{Selenium-induced defect passivation}

To assess the electronic effects of selenium alloying in CdTe we have performed correlative cathodoluminescence imaging on the bevel, on the same area as the selenium map (see Fig 1c). The map shows that at the top of the bevel, in the interdiffused CdTe region, the luminescence intensity is relatively low. However at the bottom of the bevel, in the CdSeTe region, the luminescence intensity is much brighter, with counts 10 to 20 times higher than at the top $(150,000-300,000$ counts versus $\sim 15,000)$. This steep increase in CL signal through the depth of the absorber layer mirrors the increase in selenium concentration.

In addition to this general trend down the bevel, there are variations in CL signal around the grain structure of the absorber. Firstly, it can be seen that luminescence is lower at grain boundaries than in grain interiors. This is in line with previous CL measurements on non-alloyed CdTe, and shows that grain boundaries act as nonradiative recombination centres [20]-[23]. Secondly, brighter cathodoluminescence signals can be seen around the fringes of grains in the interdiffused CdTe region. This closely matches the distribution of selenium observed around grains in the SIMS maps (see the higher magnification images in Fig 1d and Fig 1e)).

To quantify this selenium concentration vs luminescence relationship we have exactly aligned and repixellated the SIMS and CL images so that the intensity values in each can be compared pixel-for-pixel. This alignment is relatively straightforward because of the obvious features that delineate grain boundaries in both maps (see Supplementary Figure 2b, where the SIMS and CL images have been superimposed on top of one another). The results of this comparison are shown in the scatter plot in Fig 1f. The plot shows that in regions containing $<2 \%$ selenium the CL intensity is generally less than 30,000 counts, and in regions containing $>9 \%$ selenium the CL intensity is up to 300,000 counts. Since high luminescence efficiency is indicative of low levels of defect-mediated non-radiative recombination in a semiconductor, this clear positive correlation suggests that selenium passivates a non-radiative recombination centre in the alloyed material. Moreover, we have observed this effect in multiple SEM systems and on multiple samples, including on untreated CdSeTe/CdTe devices (see Supplementary Figure 3). Together these results 

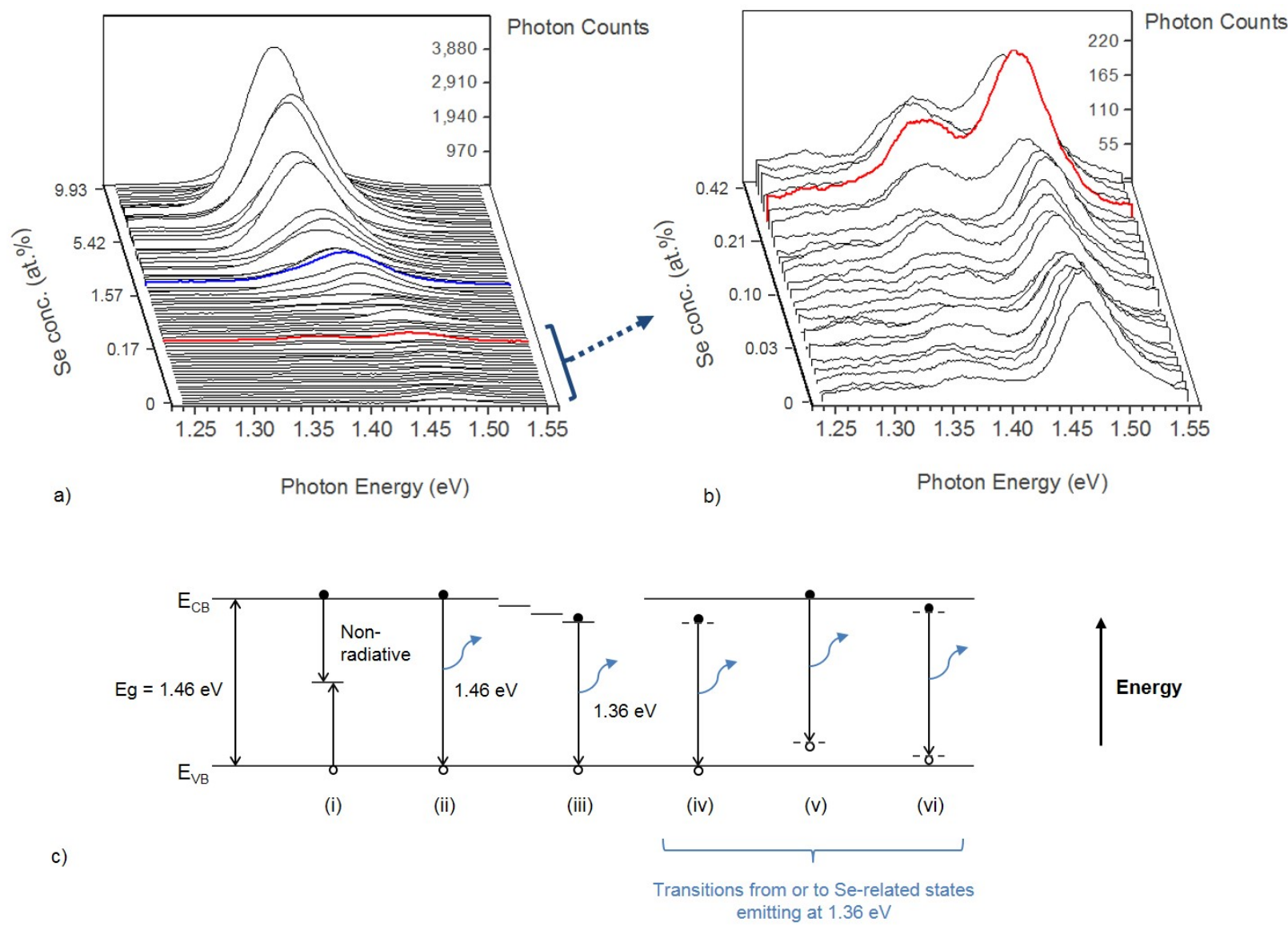

Fig 2: Hyperspectral CL imaging shows that selenium is associated with a sub-bandgap emission peak. (a) A waterfall plot of a random sample of CL spectra taken from the bevel measurement area. Curves are sorted by the concentration of selenium present in the corresponding pixel in the SIMS map. The curves highlighted in blue and red are deconvoluted in Supplementary Figures $4 \mathrm{a}$ and $4 \mathrm{~b}$ respectively. (b) A waterfall plot of the low-selenium CL spectra in (a), representing a selenium concentration range from 0 to $0.42 \%$. The curve highlighted in red is the same as that highlighted in (a) and is deconvoluted in Supplementary Figure 4b. (c) Energy band diagram showing a selection of possible electron and hole recombination channels in the material: (i) defect-mediated non-radiative recombination, (ii) radiative band-to-band recombination with emission at $1.46 \mathrm{eV}$, (iii) radiative band-to-band recombination with emission at $1.36 \mathrm{eV}$, (iv)-(vi) radiative recombination with emission at $1.36 \mathrm{eV}$, either from or to selenium-related states in the band gap. 
suggest that selenium alloying creates a passivation effect in CdTe that explains the higher open circuit voltage and improved performance of selenium-graded CdTe solar cells.

\section{Selenium related sub-bandgap states}

The cathodoluminescence image shown in Fig 1 is of the panchromatic CL signal. This means that the intensity value in each pixel is the total number of photons collected by the CL system over all wavelengths (i.e $700-$ $999 \mathrm{~nm}$ ) when the electron beam is in that region of the sample. However, important information is contained in the specific wavelengths of light that are emitted from the material, which is not displayed in the panchromatic image since it is a simple photon count. As such, in this section we build on the general picture provided by the panchromatic image by analysing the underlying spectra of wavelengths that make it up. This is possible because the CL data we collected on the bevel is hyperspectral, meaning that a full spectrum is collected at each pixel.

Figure 2a shows a waterfall plot of a representative sample of CL spectra from the panchromatic image of the bevel. To make the plot, a sample of 73 of the spectra in the hyperspectral map was selected at random for display. The curves from these pixels were then sorted by the concentration of selenium present in the corresponding pixel in the SIMS map, with the curves with the highest selenium content at the back of the plot and the lowest at the front.

The data shows a steep increase in CL intensity as the selenium concentration in the corresponding pixel increases. In addition, it shows a shift of the emission peak towards lower energies with increasing selenium content. This shift is expected, since it is known that increasing the selenium concentration initially decreases the bandgap of CdSeTe alloys due to a bandgap 'bowing' effect [7], [24] (this effect is explored in more detail in the "Selenium-induced bandgap gradients" section, where we map the effective bandgap of CdSeTe material over the bevel measurement area).

In the curves with a low selenium concentration (0-0.42 at $\%)$ plotted in Fig $2 \mathrm{~b}$ we observe two distinct emission peaks: one at $\sim 1.46 \mathrm{eV}$, which we attribute to band-to-band recombination, and a sub-bandgap peak at $\sim 1.36 \mathrm{eV}$. The intensity of the sub-gap peak increases with selenium content, indicating that selenium creates states within the bandgap when present in the CdTe in low concentrations. Possible transitions from or to such selenium-related states are depicted in Fig 2c. While the intensity of the sub-gap peak increases with selenium 
content, it is noted that that this does not affect the intensity and position of the band-to-band recombination peak. This is more clearly shown in the deconvoluted CL spectra in Supplementary Figure 4a and 4b, where the highlighted curves in the waterfall plots are analysed). It is also noted that the emission tail of the band-toband recombination peak can make a contribution to the intensity of the $1.36 \mathrm{eV}$ peak (see Supplementary Figure 4).

\section{Impact of selenium concentration on diffusion lengths}

The luminescence data presented here shows that higher levels of selenium in the CdTe material lead to an increased CL signal intensity. This suggests that selenium passivates a defect in bulk CdTe, decreasing nonradiative recombination and increasing cell performance. To confirm this, we can use CL to estimate local diffusion lengths in the material. This is done by analysing how the CL intensity varies in proximity to grain boundaries. An example of this analysis is shown in figure 3a. Here we take a line profile of the selenium and CL signals across a grain boundary in the interdiffused CdTe region of the cell, as shown in the inset. The profile shows that the selenium levels in grain (i) are higher than grain (ii), at 1 at $\%$ compared to $\sim 0.03$ at $\%$ respectively, resulting in a step in selenium concentration across the grain boundary. While there is some variation in the selenium concentration in grain (i) the profile is uniform on the length scale of the CL analytical volume, which is $\sim 1 \mu \mathrm{m}$.

Along with the change in selenium concentrations, the figure shows that there is clear asymmetry in the intensity of the CL profiles either side of the boundary. In the grain with higher selenium concentration, the total (panchromatic) CL signal plateaus at a distance $>1 \mu \mathrm{m}$ from the grain boundary (referred to from now on as the 'plateau distance', and shown by red shading in the Fig 3a). This compares to $\sim 0.7 \mu \mathrm{m}$ in the lowselenium grain. This is indicative of a greater diffusion length in the high-selenium grain because here, even when the electron beam is up to a micron away from the grain boundary, generated carriers experience the effects of the boundary (i.e. they are able to travel the $\sim 1 \mu \mathrm{m}$ distance to the boundary and recombine there, quenching the CL signal compared to its maximum level at the plateau). Calculated diffusion lengths from the profiles back this up, with the diffusion length in the $\sim 1$ at $\%$ selenium-containing grain being $0.25 \mu \mathrm{m}$, compared to $0.14 \mu \mathrm{m}$ in the low-selenium grain (it should be noted that calculations of diffusion lengths are approximate since the model assumes that the grain boundary is a free surface where carriers generated in one 
grain cannot diffuse past the grain boundary into the adjacent grain [25], [26]. In addition, states on the bevelled surface may artificially decrease minority carrier diffusion lengths).

This kind of analysis was performed in two other regions with a similar step in selenium concentration across grain boundaries, with the results shown in table 1 . The data shows that diffusion lengths in the grains with higher selenium concentration are consistently longer than in the low-selenium grains (note that grain boundaries were only chosen for this analysis if they had a step-change in selenium concentration across the boundary, as well as no other grain boundaries nearby that could influence the CL profile).

To gain further insight into why the diffusion lengths differ, we can assess the relative contributions to total CL signal variations of the band-to-band peak at $\sim 1.46 \mathrm{eV}$ and the selenium-related sub-gap peak at $\sim 1.36 \mathrm{eV}$ (Fig 3 a). The $1.43-1.49 \mathrm{eV}$ line in the figure plots the variation in CL counts within just that spectral window (shown by the right-hand grey region in Fig 3b). This captures intensity variations in just the band-to-band peak, and shows that variations in band-to-band transitions are symmetric either side of the grain boundary, despite the differences in selenium content (the plateau distance is $\sim 0.7 \mu \mathrm{m}$ on both sides of the GB). However, variations in the CL intensity in the 1.34 to $1.40 \mathrm{eV}$ window, where the $1.36 \mathrm{eV}$ selenium-related peak falls, are not symmetric across the boundary. The plateau distance for this energy range in the high-selenium grain is $>1 \mu \mathrm{m}$, compared to $\sim 0.65 \mu \mathrm{m}$ in the lowselenium grain. The $1-\mu \mathrm{m}$ long plateau distance in the sub-gap peak is therefore the reason for the similarly long plateau distance observed in the total panchromatic CL curve. This demonstrates that the long lifetime of seleniumrelated transitions enables the longer diffusion lengths in regions with higher selenium content. In addition, the symmetry of the band-edge luminescence profile $(1.43-1.49 \mathrm{eV})$ shows that any field-effects due to the stepped selenium profile across the grain boundary have not influenced the CL profiles, since the effect of an electric field is to weaken CL emission at all wavelengths. Therefore, any asymmetry in the field due to the selenium step would have caused asymmetry in the $1.43-1.49 \mathrm{eV}$ profile. 


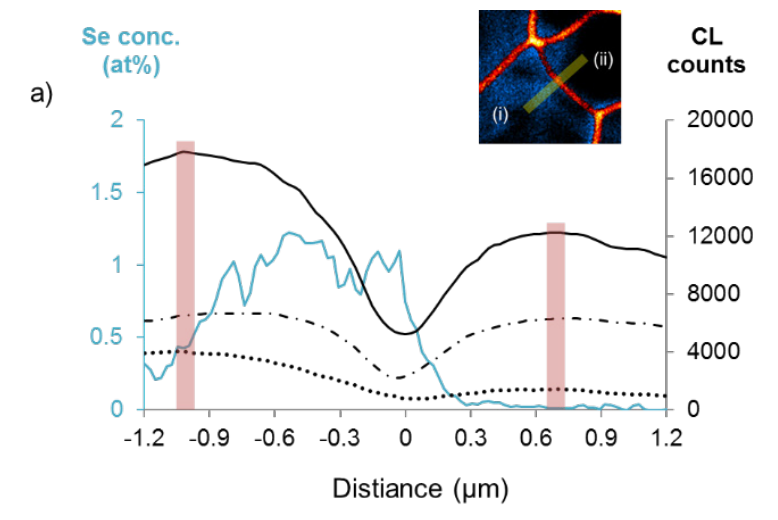

- Se conc. (at \%) - Total CL

$\cdots \cdot \operatorname{CL}(1.34-1.40 \mathrm{eV}) \quad \cdots \cdot \mathrm{CL}(1.43-1.49 \mathrm{eV})$
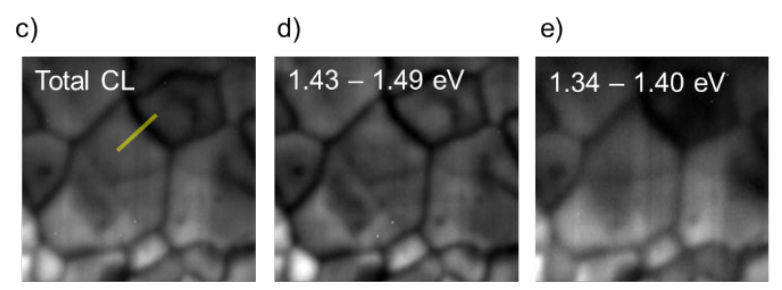

b)

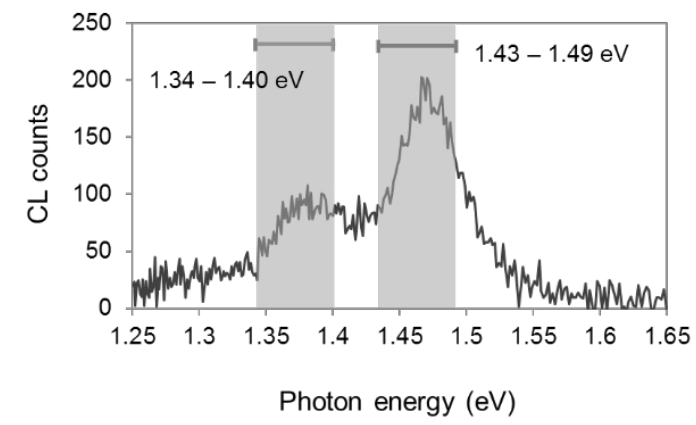

f)

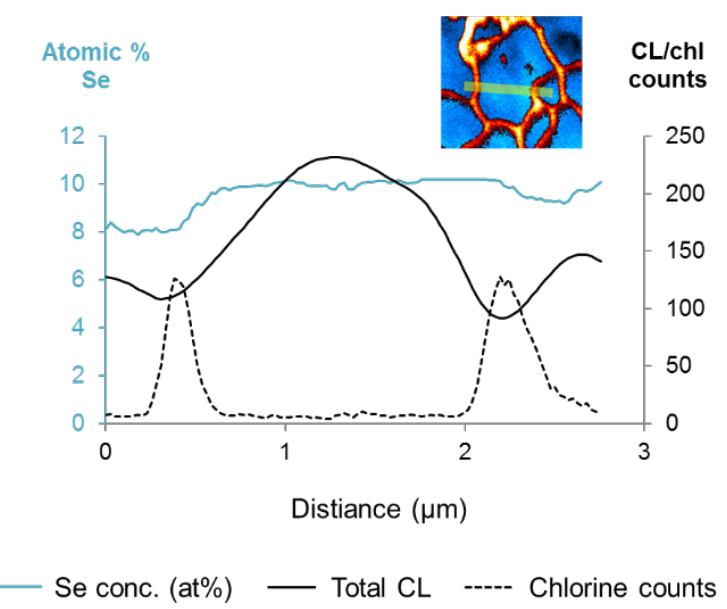

Fig 3: CdTe diffusion lengths improved by selenium alloying. (a) Profile plot of the selenium concentration (blue line, plotted on the left y-axis) and CL intensities (black lines, plotted on the right y-axis) across a grain boundary in the interdiffused CdTe region at the top of the bevel. The exact profile area is shown by the yellow line in the inset (red in the inset is the chlorine SIMS signal, blue is the selenium SIMS signal, and (i) and (ii) indicate the grains with high- and low- selenium concentrations respectively). The solid, dashed, and dot-dashed black lines show the profile of the total, $1.43-1.49 \mathrm{eV}$, and $1.34-1.40 \mathrm{eV}$ CL signals respectively (see legend). Red shading indicates the 'plateau distance' for the total CL signal on either side of the grain boundary i.e. the distance from the grain boundary, at $0 \mu \mathrm{m}$, to where the CL signal plateaus. (b) Plot of the average CL spectrum for the area analysed in panels $(c-e)$, with shading showing the spectral windows used in the analysis. (c) Map of total panchromatic CL signal in a region at the top of the bevel, with the yellow line showing the region where the profile in (a) was taken. (d) Map of CL counts in the $1.43-1.49 \mathrm{eV}$ spectral window shown by the shading in (b). (e) Map of CL counts in the $1.34-1.40 \mathrm{eV}$ spectral window shown in (b). (f) Profile plot of the selenium concentration (blue line, plotted on 
the left y-axis), chlorine SIMS counts (dashed black line, plotted on the right y-axis), and total CL intensity (solid black line, plotted on the right y-axis) across the high-selenium CdSeTe region shown in the inset (red in the inset is the SIMS chlorine signal and cyan is the SIMS selenium signal). Chlorine signal intensity is included in the plot to show the position of the grain boundaries. Note that the profile is from a region with a relatively uniform selenium concentration so as not to influence the shape of the CL profile. 
Mapping variations in CL counts in these different energy windows shows that the trend for longer plateau distances in the $1.34-1.40 \mathrm{eV}$ energy window vs the $1.43-1.49 \mathrm{eV}$ window is present across large regions of the bevel measurement area (see Figs 3c - 3e). For instance, sharp image contrast is seen in the band-to-band transitions map in Fig 3d, indicating steep V-shaped drops in CL signal across grain boundaries and therefore short plateau distances. However, when the sub-gap energy window is mapped (Fig 3e), the contrast in the image is low. This means that signal variations across grain boundaries in the image have a shallower V-shape, indicating larger plateau distances, as demonstrated by the $1.34-1.40 \mathrm{eV}$ line profile in Fig $3 \mathrm{a}$.

The diffusion length analysis has only been performed on the upper part of the bevel where the concentration of selenium is low compared to the CdSeTe region at the bottom of the bevel. This is because in the CdSeTe region there are no obvious plateaus in the CL signal within the grain interiors, making it difficult to perform either a rough 'plateau distance' analysis as done in Fig 3a, or to calculate diffusion lengths. However, this in itself is an indication of long diffusion lengths in the region, since the signal does not plateau even in large grains, such as the 2-micron diameter grain shown in Fig 3f. These results provide further evidence that selenium passivates bulk defects in CdSeTe alloys, even at very low alloying fractions.

\section{Selenium-induced bandgap gradients}

The initial purpose of alloying selenium into the front of CdTe solar cell absorbers was to decrease the material bandgap and therefore increase absorption in the low-energy part of the visible spectrum. However, very little research has been published quantifying the effects of selenium on the bandgap 

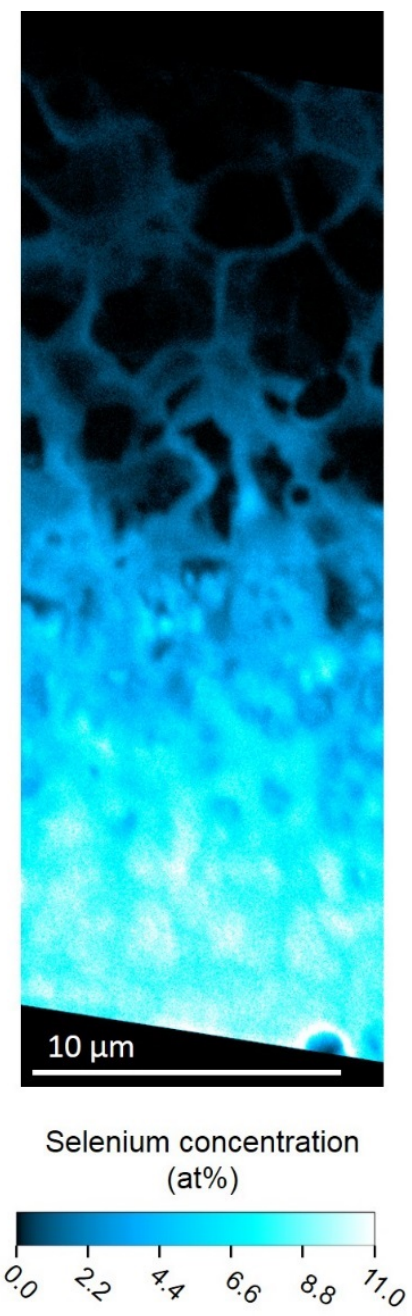

a)

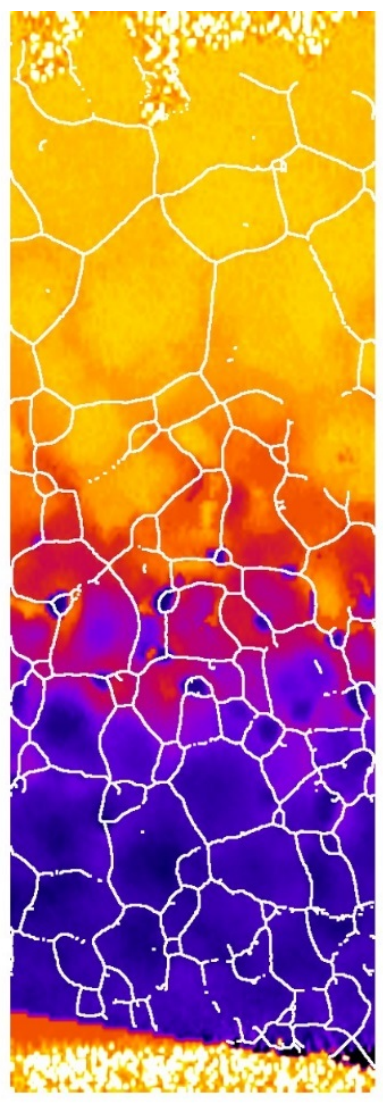

Effective bandgap

$(\mathrm{eV})$

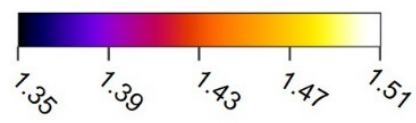

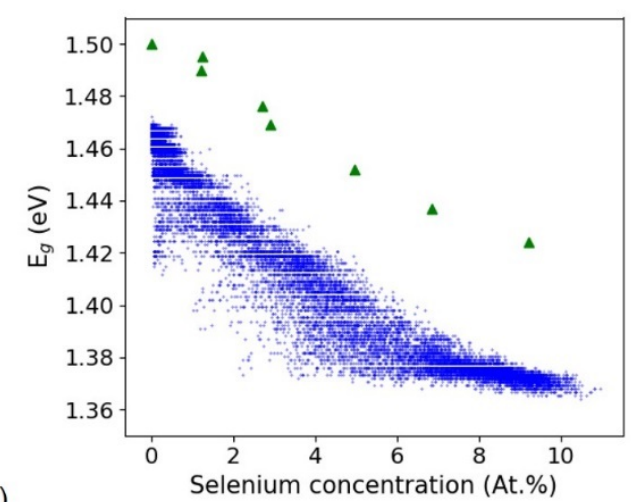

c)

d)

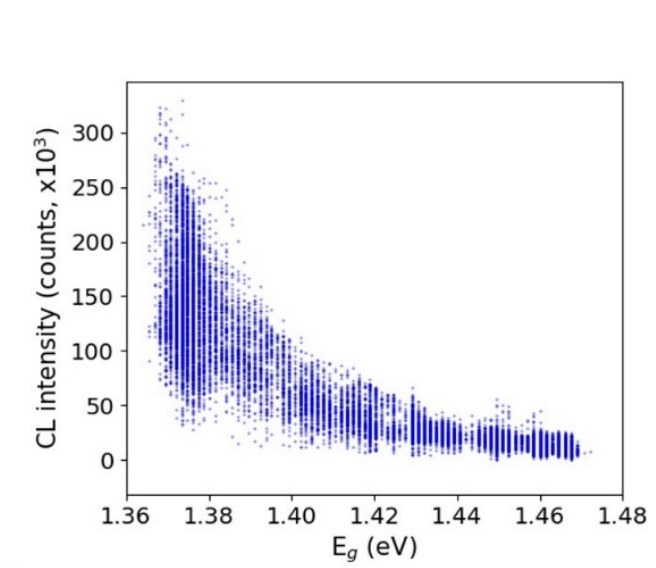

b)

Fig 4: Mapping selenium-induced bandgap changes in the absorber. (a) SIMS map of the selenium concentration on the bevel surface, as seen in figure 1 but without the skeletonised chlorine signal overlay. Darker blue corresponds to lower selenium concentrations and brighter cyan/white corresponds to higher concentrations, towards 10 at\% (see colour bar). (b) Map of the peak emission energy of the CL (i.e. the effective band gap of the material) on the same area as (a), with a skeletonised chlorine signal overlay and with pixel dimensions matched to the SIMS map. This enables pixel-for-pixel comparison of the selenium concentration and the effective band gap at each point on the bevel surface, as shown in the scatter plot in (c). Yellow/orange corresponds to a higher effective bandgap of $\sim 1.47 \mathrm{eV}$ and purple corresponds to a lower effective bandgap of $\sim 1.37 \mathrm{eV}$ (see colour bar). (c) (Blue) scatter plot of the effective bandgap vs selenium concentration at each point/pixel on the bevel, and (green) plot of the band gap vs selenium concentration reported in [7]. (d) Scatter plot of the effective bandgap vs 
panchromatic CL signal at each point on the bevel. Regions containing voids were omitted from the analysis in producing the scatter plot (voids can be seen as white spots mid-way down the bevel in the CL map in Fig 1c). 
of CdTe [7]. In this section we map the effective bandgap of CdSeTe material at high resolution over the bevel measurement area by tracking the photon energy of the dominant emission in each pixel in the CL. Fig 4a shows a SIMS map of the selenium distribution on the bevel surface, as seen in Fig 1b. Alongside this in fig $4 \mathrm{~b}$ is a map of the peak CL photon emission energy (i.e. effective bandgap), taken on the same area as the SIMS. The map shows that at the top of the bevel, towards the back contact of the device, the effective bandgap of the absorber is $\sim 1.46$ $\mathrm{eV}$ (yellow). This is typical for non-alloyed CdTe [7]. Further down the bevel, there is a steady decrease in the effective bandgap down to $\sim 1.36 \mathrm{eV}$ in the CdSeTe region (purple). This mirrors the grading of the selenium concentration and will form a built-in field through the depth of the device.

In Fig $4 \mathrm{c}$ we have plotted a scatter graph of selenium concentration against effective bandgap for each pixel in the two images, in the same way as performed previously for the total CL intensity scatter plot (Fig 1f). The plot has a classic bowing shape with an initial, steeper decrease in bandgap followed by a slight levelling off towards $10 \mathrm{at} \%$ selenium. This is consistent with the data points plotted in green (replotted from [7]), which show bandgap vs composition for untreated CdSeTe films deposited on glass using the same deposition system as used in this study. For these films, composition was obtained using energy-dispersive x-ray spectroscopy (EDX) measurements and the bandgap was extracted from absorption edge Tauc plots. Although the two curves are well-matched, the curve produced with the CL data is shifted down by $\sim 0.03 \mathrm{eV}$. This may be due to the influence of excitonic peaks in the emission spectra, which would lower the energy of the luminescence peak compared to the true bandgap [27]. In addition, the $\mathrm{CL}$ is measured in a $\mathrm{CdCl} 2$ treated film incorporated into a working device, as opposed to untreated CdSeTe films on a glass substrate, so intentional absorber impurities such as chlorine may slightly alter the material bandgap.

Table 1: Diffusion lengths. Calculated diffusion lengths either side of three grain boundaries in the interdiffused CdTe region. Across each boundary there is a step in the selenium concentration. Grain boundary 1 is shown in Fig 3a. 


\begin{tabular}{|c|l|l|l|}
\hline Grain Boundary & Side & $\begin{array}{l}\text { Se Content } \\
(\mathrm{at} \%)\end{array}$ & $\begin{array}{l}\text { Diffusion Length } \\
(\mu \mathrm{m})\end{array}$ \\
\hline \multirow{2}{*}{1} & Se-poor side & $0.03 \%$ & 0.143 \\
\cline { 2 - 4 } & Se-rich side & $0.94 \%$ & 0.246 \\
\hline \multirow{2}{*}{2} & Se-poor side & $0.14 \%$ & 0.163 \\
\cline { 2 - 4 } & Se-rich side & $0.90 \%$ & 0.197 \\
\hline \multirow{2}{*}{3} & Se-poor side & $0.02 \%$ & 0.118 \\
\cline { 2 - 4 } & Se-rich side & $0.58 \%$ & 0.203 \\
\hline
\end{tabular}

As well as a grading through the depth of the absorber the map in Fig $4 \mathrm{~b}$ shows variations in the effective bandgap around grain boundaries in the material. At the top of the bevel, in the interdiffused CdTe region, there is a decrease in the effective bandgap around grain boundaries and grain fringes (darker orange) vs the grain interiors (lighter yellow). This matches the positioning of selenium seen in the SIMS maps, and corresponds to a decrease in bandgap of $\sim 0.01 \mathrm{eV}$ in these regions. However, at the bottom of the bevel, in the CdSeTe region, there is an increase in effective bandgap around grain boundaries (lighter purple). This tracks the decrease in the selenium content seen at grain boundaries in the SIMS maps in this region.

Fig $4 \mathrm{~d}$ shows a scatter plot of effective bandgap versus total CL signal at each point on the bevel. The plot shows an initially shallow, and then steep rise in material luminescence efficiency with decreasing bandgap. This means that, for instance, decreasing the bandgap from $1.46 \mathrm{eV}$ to $1.42 \mathrm{eV}$ gives a much smaller increase in defect passivation than an equal step from $1.42 \mathrm{eV}$ to $1.38 \mathrm{eV}$. This information will be useful to device designers and fabricators when deciding on, or modelling the optimum selenium grading profile.

\section{Discussion}

Remarkable progress has been made in cadmium telluride photovoltaics despite the lack of a full, fundamental understanding of absorber layer material properties. This is especially true of the record-breaking selenium-graded devices. In this work, by means of correlative cathodoluminescence and SIMS measurements, we have shown that selenium alloying enables high luminescence efficiency in alloyed CdTe, suggesting that it passivates defects in 
both $\mathrm{CdCl}_{2}$-treated and untreated bulk CdTe. Further evidence for this passivation effect is provided by an analysis of CL signal variations across grain boundaries, which shows longer carrier diffusion lengths in alloyed regions with higher selenium content. Hyperspectral CL imaging shows that selenium not only shifts the band-to-band emission peak from $\sim 1.46 \mathrm{eV}$ to $\sim 1.36 \mathrm{eV}$, creating a bandgap gradient and built-in field across the absorber, but it also creates radiative sub-gap states at $\sim 1.36 \mathrm{eV}$, which is the same energy as the lowest point on the bandgap bowing curve. The high-resolution SIMS measurements show that during the $\mathrm{CdCl}_{2}$ heat treatment process selenium diffuses from the CdSeTe into the CdTe, primarily along grain boundaries but with some out-diffusion from grain boundaries into grain interiors. This causes an excess of selenium at grain boundaries in the CdTe layer that decreases the material bandgap in those regions. In addition, it causes a selenium deficiency at grain boundaries in the CdSeTe layer, resulting in higher band gaps in these regions relative to the grain interiors. This will have unknown effects on carrier transport around grain boundaries in selenium-graded devices.

These results show that in non-alloyed CdTe there are deep-level defects in the bulk material that act as recombination centres and limit device efficiency, even following the $\mathrm{CdCl} 2$ treatment. Potential candidates for these defects are cadmium vacancies (VCd) and tellurium-on-cadmium antisites (TeCd), which Density Functional Theory (DFT) modelling has shown act as harmful recombination centres [28], [29]. We suggest that these defects can be passivated or made less likely to form when selenium is present, with an increasing passivation effect for concentrations of selenium up to $10-11$ at $\%$. A slight levelling out of the passivation effect towards $10-11 \%$ suggests this may be close to the optimum alloying concentration in terms of absorber material quality.

This defect passivation explains the remarkable performance of CdSeTe devices. However, current high efficiency devices only have selenium in significant concentrations at the very front of the device, leaving much of the back of the absorber layer unpassivated. This means that extending the selenium profile further towards the back of the device, whilst maintaining a concentration gradient and built-in field, will passivate more defects at the back of the absorber and might improve efficiencies further. In addition, we note that the energy of the sub-gap selenium-related emission $(\sim 1.36 \mathrm{eV})$ is similar to the lowest achievable effective bandgap energy in the selenium-rich layer, which also shows the highest luminescence intensity. This indicates that all these effects can be related to a common underlying mechanism.

In summary, in this work we have correlated variations in the electronic properties of selenium-alloyed CdTe with local variations in the selenium concentration. We find a strong correlation between selenium concentration, high 
material luminescence efficiency, sub-gap transitions at $1.36 \mathrm{eV}$, and longer diffusion lengths, all at the sub-micron scale. This indicates that selenium passivates defects present in bulk CdTe, and provides an explanation for the remarkable performance of selenium-alloyed CdTe devices. In addition, the results provide crucial insights into the fundamental electronic behaviour of selenium alloyed $\mathrm{CdTe}$, which could unlock further improvements in the photovoltaic performance of CdSeTe solar cells.

\section{Acknowledgements}

The authors at Loughborough University are grateful to the EPSRC CDT in New and Sustainable PV for providing TF with a studentship, to RCUK for funding through the EPSRC Supergen SuperSolar Hub (EP/J017361/1), and to the Loughborough Materials Characterisation Centre (LMCC) for use of equipment. The authors at CSU would like to acknowledge support from NSF AIR, NSF I/UCRC and DOE- SIPS programs. The work at Colorado State University was supported by NSF award 1540007, NSF PFI:AIR-RA program 1538733 and U.S. Department of Energy Small Innovative Projects in Solar (SIPS), DE-EE0008177. KL acknowledges support from EPSRC grant M018237/1.

\section{Methods}

Cell Fabrication and Electrical Testing. The two types of cells used in this study were deposited on TEC 10 glass substrates supplied by NSG Pilkington ltd. The substrates consist of $3 \mathrm{~mm}$ of soda lime glass coated with a $\sim 400$ $\mathrm{nm}$ layer of fluorine-doped tin oxide, which acts as the transparent conducting oxide (TCO). The other layers of the cells were then fabricated as follows: $100 \mathrm{~nm}$ of $\mathrm{MgZnO}$ was deposited on the TCOs by magnetron sputtering, forming the buffer/window layer of the devices. This was followed by $1.5-2 \mu \mathrm{m}$ of CdSeTe, deposited with the substrates held at $\sim 420{ }^{\circ} \mathrm{C}$ and from a source containing $40 \%$ CdSe at $575{ }^{\circ} \mathrm{C}$. A $\sim 3.5 \mu$ m layer of CdTe was then deposited on top of the CdSeTe with the substrates held at $500{ }^{\circ} \mathrm{C}$ and the CdTe source at $555^{\circ} \mathrm{C}$. A cadmium chloride $\left(\mathrm{CdCl}_{2}\right)$ activation treatment was then performed on one of the substrates. This involved sublimation of a $\mathrm{CdCl} 2$ vapour onto the back surface of the substrate whilst it was maintained at $430{ }^{\circ} \mathrm{C}$ for 600 seconds, followed by a $180{ }^{\circ} \mathrm{C}$ cooling step with the substrate removed from the vapour. Both device stacks then received a copper doping treatment whereby copper chloride was deposited on the back surface of the CdTe for 110 seconds whilst the substrate was held at 
$\sim 140{ }^{\circ} \mathrm{C}$. This was followed by an anneal in vacuum at $220^{\circ} \mathrm{C}$ for 220 seconds to drive copper into the device. Finally, a $\sim 30 \mathrm{~nm}$ Te film was evaporated on to the back of the CdTe to improve the back contact. At this stage the two substrates were split in half, with one half of each substrate undergoing contacting and performance testing, and the other half left bare for materials characterisation.

For cell contacting a layer of carbon and nickel paint in a polymer binder was sprayed on the back of the device stack, forming the back electrode of the device. This was then masked and sand- blasted to delineate 10 separate cells of area $0.55 \mathrm{~cm}^{2}$. The devices were then tested for electrical performance using current density vs voltage measurements using an AM1.5 spectrum. An ABET Technologies 10,500 solar simulator with uniform illumination accessory was used to illuminate the devices for measurements. The lamp used for illumination is an ozone free DC xenon arc lamp that produces 1 Sun power output over a $35 \mathrm{~mm}$ diameter field and meets ASTM, IEC and JIS Class A AM1.5G output requirements. Current density-voltage curves were generated based on electrical measurements performed using a Keithley 2420 SourceMeter controlled by a LabView program. Shortcircuit current density was calibrated to CdTe cells measured by NREL. Device areas were measured using a webcam that took an image of a backlit solar cell and counted the pixels below certain brightness. Both the light intensity and area were calibrated for each set of measurements. The cells were contacted by a fixture of springloaded gold pins that provided a 4-point connection and collect current from all around the front contact of the device. The setup accurately measures the J- V parameters and the agreement of these measurements with an externally certified photovoltaic device has been shown in previous work [18].

TEM. The specimen foil for scanning transmission electron microscopy (STEM) was prepared using an FEI focused ion beam (FIB) dual beam system using a standard in-situ lift out method [30]. STEM imaging was performed using a FEI Tecnai F20 S/TEM equipped with Gatan Bright and Dark field STEM detectors, Fischione High Angle Annular Dark Field (HAADF) STEM detector and an Oxford Instruments X-Max $80 \mathrm{~mm}^{2}$ windowless energy-dispersive spectrometer (EDX). STEM imaging was performed at $200 \mathrm{kV}$ with a camera length of $100 \mathrm{~mm}$ and condenser aperture size of $70 \mu \mathrm{m}$.

Cathodoluminescence. To present an extended cross-section for cathodoluminescence (CL) and SIMS characterisation, a bevel was milled through the $\mathrm{CdCl} 2$ treated device stack at an angle of 7 degrees to the 
horizontal using a $30 \mathrm{keV}$ gallium focussed ion beam in an FEI Nova 600 Nanolab Dual Beam scanning electron microscope(SEM). A platinum over-layer was deposited on the back surface of the CdTe to homogenize the FIB milling of the bevel. Hyperspectral CL characterisation was carried-out at room temperature in a Hitachi SU-70 scanning electron microscope (SEM). During the measurement a $7 \mathrm{keV}$ beam was rastered across an area on the bevel surface. Luminescence emitted from the sample surface was collected by a parabolic mirror and fed through a diffraction grating to a Gatan MonoCL system for CL detection. This resulted in a $102 \times 282$ pixel image, with each pixel containing a full CL spectrum. At $7 \mathrm{keV}$, CASINO Monte Carlo simulations show that most of the carriers excited by the beam in CdTe (75\%) are generated within a tear-drop shaped volume that extends $200 \mathrm{~nm}$ below the sample surface and has a diameter of $\sim 200 \mathrm{~nm}$ (note that results are for simulations performed at 7.5 $\mathrm{keV)}[21]$.

NanoSIMS. Following the CL, high resolution elemental mapping was performed on the same area of the bevel surface using a Cameca secondary ion mass spectrometer (NanoSIMS 50) with a $16 \mathrm{keV} \mathrm{Cs}^{+}$primary beam. The diameter of the D1 aperture was set to $100 \mu \mathrm{m}$ (D1-4). Entrance and Aperture slits are 50 x $220 \mu \mathrm{m}$ (ES-1) and open, respectively. During the measurement, a $0.5-1 \mathrm{pA} \mathrm{Cs}^{+}$primary beam with a nominal diameter of $60 \mathrm{~nm}$ was rastered over the measurement area and sputtered secondary ions analysed with a double-focused mass spectrometer. The raster size was 25 x $25 \mu \mathrm{m}(512$ x 512 pixels $)$ and the dwell time was $500 \mu$ s per pixel. Masses analysed were $35 \mathrm{Cl}^{-}$and $80 \mathrm{Se}^{-}$, giving high resolution images of distributions of chlorine and selenium in the measured area. The scan was repeated 20 times giving 20 stacked images of the distributions of each element and sputtering a total depth of $\sim 200 \mathrm{~nm}$ below the bevel surface. Images used in the figures are a sum of each stack of 20 images. The 'auto-track' feature in ImageJ was used to correct a small amount of image drift before the images were summed. Summing the images ensures that the information depth of the SIMS, at $\sim 200 \mathrm{~nm}$, is similar to the CL information depth (100- $200 \mathrm{~nm}$ excluding carrier drift/diffusion). EDX measurements were taken on the bevel at to calibrate the selenium counts obtained in the NanoSIMS measurements (beam energy $20 \mathrm{keV}$ ). They showed that the average selenium concentrations in the CdSeTe region were $8.9 \%$. This value could then be used to calibrate the average selenium counts over the same region/area given by the NanoSIMS.

Image processing. Some image manipulations were required to allow exact alignment of the pixels of the CL and 
SIMS images on the same area of the bevel. First, the SIMS maps were rotated to match the orientation of the CL map. The CL map was then scaled by $+13.5 \%$ in the Y-direction, i.e. height (this was necessary because of some drift in the raster of the electron beam in the CL measurements, creating a slight distortion that shortened the image). The two sets of images could now be superimposed on top of one another with exact matching of grain boundary features in each (grain boundaries are delineated clearly by the chlorine signal in the SIMS and darker valleys in the CL). At this point the images are matched in everything but the pixel size (the SIMS images are higher resolution). For the pixel-for-pixel comparisons given in the scatter graphs, the pixel size of the SIMS was increased to match the $\mathrm{CL}$, giving images of $102 \times 282$ pixels that could be directly compared. For the maps used in the figures, the resolution of the SIMS image was maintained ( 389 x 1152 pixels). Image rotation, scaling, and repixellation were performed using ImageJ.

\section{Additional information}

Correspondence and requests for materials should be addressed to J.M.W

\section{Data Availability Statement}

The data that supports the plots within this paper and other findings of this study are available in the repository at Loughborough University or from the corresponding author upon reasonable request.

\section{$\underline{\text { Author contributions }}$}

This work was conceived of by TF, and planned with JW, KL, CG, and BM. The cells were made by AM, with the assistance of KB and WS. TEM was performed by AA. Samples were prepared for CL and SIMS characterisation by TF. CL measurements were carried out by BM. SIMS characterisation was performed by KL, with assistance from CG. Data analysis was performed by TF and LW. The manuscript was written by TF, with help from JB and JW.

\section{Competing interests}

The authors declare no competing financial or non-financial interests. 


\section{References}

[1] M. Gloeckler, I. Sankin, and Z. Zhao, "CdTe Solar Cells at the Threshold to 20\% Efficiency,” IEEE J. Photovoltaics, vol. 3, no. 4, pp. 1389-1393, Oct. 2013.

[2] M. A. Green, Y. Hishikawa, E. D. Dunlop, D. H. Levi, J. Hohl-Ebinger, and A. W. Y. Ho-Baillie, "Solar cell efficiency tables (version 51)," Prog. Photovoltaics Res. Appl., vol. 26, no. 1, pp. 3-12, 2018.

[3] “Lazard’s Levelised Cost of Energy Analysis - Version 11.0,” no. 11, pp. 0-21, 2017.

[4] J. Balderelli, "Public Utilities Comission of Nevada Electronic Filing," 2015.

[5] C. Hagenorf, M. Ebert, M. Raugei, D. Lincot, J. Bengoechea, and M. Rodriguez, “Cener Report: Assessment of performance, environmental, health and safety aspects of First Solar's CdTe PV technology," 2016.

[6] N. R. Paudel and Y. Yan, "Enhancing the photo-currents of CdTe thin-film solar cells in both short and long wavelength regions," Appl. Phys. Lett., vol. 105, no. 18, 2014.

[7] D. E. Swanson, J. R. Sites, and W. S. Sampath, “Co-sublimation of CdSe x Te 1 À x layers for CdTe solar cells," Sol. Energy Mater. Sol. Cells, vol. 159, pp. 389-394, 2017.

[8] J. D. Poplawsky, W. Guo, N. Paudel, A. Ng, K. More, D. Leonard, and Y. Yan, "Structural and compositional dependence of the CdTexSe1-x alloy layer photoactivity in CdTe-based solar cells," Nature Communications, vol. 7. p. 12537, 2016.

[9] A. H. Munshi, J. Kephart, A. Abbas, J. Raguse, J. Beaudry, K. Barth, J. Sites, J. Walls, and W. Sampath, "Polycrystalline CdSeTe/CdTe Absorber Cells With 28 mA/cm 2 Short-Circuit Current," IEEE J.

Photovoltaics, vol. 8, no. 1, pp. 310-314, Jan. 2018.

[10] J. M. Kephart, A. Kindvall, D. Williams, D. Kuciauskas, P. Dippo, A. Munshi, and W. S. Sampath, “Sputter-Deposited Oxides for Interface Passivation of CdTe Photovoltaics," IEEE J. Photovoltaics, pp. 1$7,2018$.

[11] D. Kuciauskas, J. M. Kephart, J. Moseley, W. K. Metzger, W. S. Sampath, and P. Dippo, "Recombination velocity less than $100 \mathrm{~cm} / \mathrm{s}$ at polycrystalline Al 2 O 3 /CdSeTe interfaces," Appl. Phys. Lett., vol. 112, no. 26, p. 263901, Jun. 2018.

[12] A. H. Munshi, A. H. Danielson, T. Fiducia, A. Abbas, A. P. Nicolson, L. Kurt, J. M. Walls, and W. S. Sampath, "Electron Reflector Behavior of CdTe for CdSeTe Absorber Devices," Sol. Energy Mater. Sol. Cells, no. In-press.

[13] I. Dharmadasa, "Review of the CdCl2 Treatment Used in CdS/CdTe Thin Film Solar Cell Development and New Evidence towards Improved Understanding," Coatings, vol. 4, no. 2, pp. 282-307, 2014.

[14] E. S. Barnard, B. Ursprung, E. Colegrove, H. R. Moutinho, N. J. Borys, B. E. Hardin, C. H. Peters, W. K. Metzger, and P. J. Schuck, “3D Lifetime Tomography Reveals How CdCl2 Improves Recombination Throughout CdTe Solar Cells," Adv. Mater., vol. 29, no. 3, 2017.

[15] T. A. M. Fiducia, K. Li, A. H. Munshi, K. Barth, W. S. Sampath, C. R. M. Grovenor, and J. M. Walls, “3D Distributions of Chlorine and Sulphur Impurities in a Thin-Film Cadmium Telluride Solar Cell," MRS Adv., 
vol. 3, no. 56, pp. 3287-3292, May 2018.

[16] A. Abbas, G. D. West, J. W. Bowers, P. Isherwood, P. M. Kaminski, B. Maniscalco, P. Rowley, J. M. Walls, K. Barricklow, W. S. Sampath, and K. L. Barth, "The effect of cadmium chloride treatment on close-spaced sublimated cadmium telluride thin-film solar cells," IEEE J. Photovoltaics, vol. 3, no. 4, pp. 1361-1366, 2013.

[17] Amit H. Munshi, "Polycrystalline CdTe Photovoltaics with Efficiency Over 18\% through Improved Absorber Passivation and Current Collection.”

[18] A. H. Munshi, J. M. Kephart, A. Abbas, A. Danielson, G. Gélinas, J.-N. Beaudry, K. L. Barth, J. M. Walls, and W. S. Sampath, "Effect of $\mathrm{CdCl} 2$ passivation treatment on microstructure and performance of CdSeTe/CdTe thin-film photovoltaic devices," Sol. Energy Mater. Sol. Cells, vol. 186, no. May, pp. 259$265,2018$.

[19] L. G. Harrison, "Influence of dislocations on diffusion kinetics in solids with particular reference to the alkali halides," Trans. Faraday Soc., vol. 57, p. 1191, 1961.

[20] H. R. Moutinho, J. Moseley, M. J. Romero, R. G. Dhere, C. S. Jiang, K. M. Jones, J. N. Duenow, Y. Yan, and M. M. Al-Jassim, "Grain boundary character and recombination properties in CdTe thin films," Conf. Rec. IEEE Photovolt. Spec. Conf., pp. 3249-3254, 2013.

[21] J. Moseley, M. M. Al-Jassim, D. Kuciauskas, H. R. Moutinho, N. Paudel, H. L. Guthrey, Y. Yan, W. K. Metzger, and R. K. Ahrenkiel, "Cathodoluminescence analysis of grain boundaries and grain interiors in thin-film CdTe," IEEE J. Photovoltaics, vol. 4, no. 6, pp. 1671-1679, 2014.

[22] J. Moseley, W. K. Metzger, H. R. Moutinho, N. Paudel, H. L. Guthrey, Y. Yan, R. K. Ahrenkiel, and M. M. Al-jassim, "Recombination by grain-boundary type in CdTe," J. Appl. Phys., vol. 118, 2015.

[23] G. Stechmann, S. Zaefferer, T. Schwarz, P. Konijnenberg, D. Raabe, C. Gretener, L. Kranz, J. Perrenoud, S. Buecheler, and A. Nath Tiwari, “A correlative investigation of grain boundary crystallography and electronic properties in CdTe thin film solar cells," Sol. Energy Mater. Sol. Cells, vol. 166, no. January, pp. 108-120, 2017.

[24] D. W. Lane, “A review of the optical band gap of thin film CdSxTe1-x," Sol. Energy Mater. Sol. Cells, vol. 90, no. 9, pp. 1169-1175, 2006.

[25] B. G. Mendis, L. Bowen, and Q. Z. Jiang, "A contactless method for measuring the recombination velocity of an individual grain boundary in thin-film photovoltaics," Appl. Phys. Lett., vol. 97, no. 9, pp. 0-3, 2010.

[26] B. G. Mendis and L. Bowen, "Cathodoluminescence measurement of grain boundary recombination velocity in vapour grown p-CdTe,” J. Phys. Conf. Ser., vol. 326, no. 1, 2011.

[27] J. Lee, N. C. Giles, D. Rajavel, and C. J. Summers, "Room-temperature band-edge photoluminescence from cadmium telluride," Phys. Rev. B, vol. 49, no. 3, p. 1668, 1994.

[28] J.-H. Yang, W.-J. Yin, J.-S. Park, J. Ma, and S.-H. Wei, "Review on first-principles study of defect properties of CdTe as a solar cell absorber,” Semicond. Sci. Technol., vol. 31, no. 8, p. 83002, 2016.

[29] J. H. Yang, L. Shi, L. W. Wang, and S. H. Wei, "Non-Radiative Carrier Recombination Enhanced by TwoLevel Process: A First-Principles Study,"Sci. Rep., vol. 6, no. February, pp. 1-10, 2016. 
[30] A. Abbas, "The microstructure of thin film cadmium telluride photovoltaic materials," Loughborough University, 2014. 